\title{
PARTO VAGINAL DESPUÉS DE UNA CESÁREA PREVIA, HOSPITAL SAN JUAN DE DIOS, CALI (COLOMBIA). ESTUDIO DE COHORTE
}

\section{A cohort study of vaginal birth following prior cesarean section in the San Juan de Dios hospital, Cali, Colombia}

Olga Lucía Cuero-Vidal, M.D. *, Carlos Andrés Clavijo-Prado, M.D. **

Recibido: noviembre 11/10 - Aceptado: mayo 19/11

\section{RESUMEN}

Objetivo: determinar la incidencia de parto vaginal después de una cesárea previa y evaluar la capacidad predictiva del puntaje predictor de parto vaginal descrito por Flamm en 1997.

Materiales y métodos: estudio de cohorte retrospectivo de mujeres con antecedente de cesárea y embarazo de al menos 37 semanas, que consultaron al Hospital San Juan de Dios de Cali (HSJD) entre mayo 30 de 2007 y mayo 30 de 2008. Se calculó la tasa de parto vaginal y se evaluó la sensibilidad, especificidad, valores predictivos positivo (VPP) y negativo (VPN) y las razones de verosimilitud positiva (RVP) y negativa (RVN) del puntaje predictor de parto vaginal después de una cesárea cuando los valores de estos son de 4 o más y de 8 o más.

Resultados: la proporción de parto vaginal en este grupo fue del 20,3\%. El 36\% de las gestantes con puntajes entre 4 y 7 tuvieron parto vaginal y el $83 \%$

* Médica de la Universidad del Valle, especialista en Ginecología y Obstetricia de la Universidad de Antioquia, candidata al título de Magíster en Epidemiología de la Universidad del Valle. Ginecóloga Obstetra del Hospital San Juan de Dios y Docente de la Facultad de Medicina de la Universidad Santiago de Cali. Cali (Colombia). Correo electrónico: linary67@ hotmail.com

** Médico de la Universidad Santiago de Cali, Médico de SALUDCOOP - EPS, Médico Asistencial en la Unidad de Cuidado Coronario, Corporación Universitaria Comfenalco, Universidad Libre. Cali (Colombia).Correo electrónico: carlosandresclavijo@hotmail.com de las gestantes con 8 o más puntos. Con un punto de corte $\geq 4$ puntos la sensibilidad fue de $85,7 \%$, la especificidad 69,1\%, VPP 41,3\% y VPN 95\%, RVP 2,8 y RVN 0,2. Si el punto de corte fue $\geq 8$ la sensibilidad fue de $83,3 \%$, la especificidad $82,6 \%$, VPP 17,9\% y VPN 99,1\%, RVP 4,8 y RVN 0,2.

Conclusión: la frecuencia de parto vaginal posterior a cesárea es del 20\%. Las gestantes con puntajes menores de 4 tuvieron una baja probabilidad de tener un parto vaginal.

Palabras clave: cesárea previa, parto vaginal, factor predictor.

\section{SUMMARY}

Objective: determining the incidence of vaginal birth following a prior cesarean section and evaluating the predictive ability of the vaginal birth predictor score on the admission scoring system described by Flamm in 1997.

Materials and methods: this was a retrospective cohort study of women having undergone a prior cesarean section and then a pregnancy lasting at least 37 weeks who had consulted at the San Juan de Dios hospital in Cali (HSJD) between May $30^{\text {th }}$ 2007 and May30 ${ }^{\text {th }}$ 2008. The vaginal birth rate was calculated and sensitivity, specificity, positive (PPV) and negative predictive values (NPV) were evaluated, as were positive (RVP) and negative 
verisimilitude ratios $(\mathrm{RVN})$ regarding vaginal birth predictor score following a cesarean when their scores were 4 or more and 8 or more.

Results: there were 20.3\% vaginal births in this group; $36 \%$ of the pregnant women having scores between 4 and 7 had undergone vaginal birth and $83 \%$ of the expectant mothers scoring 8 or more. A $\geq 4$ points cut-off point gave $85.7 \%$ sensitivity, 69.1\% specificity, 41.3\% Positive Predictive Value (PPV) and 95\% Negative Predictive Value (NPV) and 2.8 Positive likelihood ratio P LR(+) and 0.2 Negative likelihood ratio (NLR). If cut-off point were $\geq 8$, then this gave $83.3 \%$ sensitivity, $82.6 \%$ specificity, 17.9\% PPV, 99.1\% NPV, $4.8 \mathrm{RVP}$ and 0.2 RVN.

Conclusion: vaginal birth frequency following a cesarean section was 20\%. Pregnant women having scores less than 4 had a low probability of having a vaginal birth.

Key words: prior cesarean section, vaginal birth, predictor factor.

\section{INTRODUCCIÓN}

La tasa de cesárea es variable entre países y entre instituciones. ${ }^{1,2}$ En Estados Unidos la incidencia de cesárea es del 26,1\%, en América Latina del 29\% en promedio, observándose los índices más elevados en México, Brasil, República Dominicana y Chile, los cuales oscilan entre 30\% y 39\%. ${ }^{1-5}$ En Colombia la tasa de cesárea reportada es de $27 \%$ pero llega hasta un $67 \%$ en instituciones privadas. ${ }^{1,6}$ Actualmente, se considera el parto vaginal en pacientes con cesárea previa como una alternativa segura, que no presenta un incremento en la morbimortalidad maternofetal, donde el riesgo de ruptura uterina es la principal preocupación; sin embargo, ésta se presenta en menos del $1 \%$ de los casos. ${ }^{7-12}$ Los estudios reportan que hasta un $82 \%$ de las pacientes con antecedente de cesárea podrían tener un parto vaginal. ${ }^{8-22}$

Se han descrito algunos factores que favorecen la probabilidad de parto vaginal después de una cesárea previa, entre ellos se encuentran: tener un parto vaginal previo, tener el cuello uterino borrado al momento de la admisión, no tener la misma causa que ocasionó la cesárea anterior, mujeres blancas, entre otros. ${ }^{23-28}$ En 1997, Flamm propuso un puntaje con el objetivo de predecir la probabilidad de parto vaginal después de una cesárea. El puntaje es obtenido a partir de un modelo de regresión logística que determina 5 variables a tener en cuenta: la edad materna menor a 40 años, el antecedente de parto vaginal previo, la causa de la primera cesárea, el borramiento y la dilatación al momento de la admisión (tabla 1). El autor encuentra que el aumento en el puntaje se asocia linealmente con el aumento de probabilidad de parto vaginal, y ésta varía entre un 49\% para puntajes de 0-3, y hasta un 97\% para puntajes de $8-10 .{ }^{28}$

En nuestro medio son pocos los estudios publicados sobre la frecuencia de cesárea, como también sobre las tasas de parto después de una cesárea previa; además, no se ha evaluado la capacidad predictiva de parto vaginal del puntaje predictor sugerido por Flamm. Por lo tanto, se realiza el presente estudio con el fin de determinar la incidencia de cesárea, así como estimar la frecuencia de parto vaginal después de cesárea y evaluar el puntaje predictor de parto vaginal de Flamm en gestantes que ingresaron al Hospital San Juan de Dios de Cali.

\section{MATERIALES Y MÉTODOS}

\section{Diseño}

Estudio de cohorte retrospectivo (histórico) de pacientes que asistieron entre el 30 de mayo de 2007 y el 30 de mayo de 2008, para atención de parto al Hospital San Juan de Dios de Cali (HSJD). Este hospital es uno de los centros de atención de nivel II del suroccidente colombiano y lugar de prácticas universitarias; también es considerado uno de los principales centros de remisión de pacientes obstétricas con patologías. Atiende a población que pertenece a los estratos 1,2 y 3 de la ciudad de Cali y municipios aledaños. 


\begin{tabular}{|l|l|l|}
\hline $\begin{array}{l}\text { Tabla 1. Instrumento para estimar el puntaje } \\
\text { predictor de parto vaginal. Flamm }\end{array}$ \\
\hline 1 & Edad menor de 40 & 2 puntos \\
\hline 2 & Parto vaginal previo: & \\
\hline & Antes y después de cesárea & 4 puntos \\
\hline Luego de primera cesárea & 2 puntos \\
\hline Antes de primera cesárea & 1 puntos \\
\hline 3 & Ringuno & 0 puntos \\
\hline Distinta a la falla en progreso de & 1 puntos \\
\hline parto & Borramiento al admitirse: & \\
\hline$>75 \%$ & & 1 puntos \\
\hline
\end{tabular}

En el estudio se incluyeron todas las mujeres que ingresaron al Hospital con diagnóstico de embarazo mayor o igual a 37 semanas, con dos o más embarazos y antecedente de una cesárea previa y con actividad uterina espontánea en el momento del ingreso. Se excluyeron las embarazadas con antecedente de cesárea previa con incisión corporal, diagnóstico de distocia pélvica, sufrimiento fetal agudo en el momento del ingreso, situación transversa, presentación podálica, macrosomía fetal, antecedente de endometritis posparto, no deseo de la paciente de parto vaginal, u otros factores considerados como contraindicaciones relativas y/o absolutas para parto vaginal. El protocolo fue aprobado por el comité de ética de la Universidad Santiago de Cali y la dirección del Hospital San Juan de Dios de Cali lugar de prácticas de esta universidad.

\section{Tamaño muestral}

Anualmente, se atienden en el hospital San Juan de Dios de Cali un promedio de 3600 partos al año, de acuerdo a los registros del departamento de estadística del Hospital San Juan de Dios; e ingresan aproximadamente 750 gestantes anuales con antecedente de cesárea previa. Considerando una incidencia esperada de parto vaginal después de una cesárea del 80\%, ${ }^{13-22}$ un error de estimación del 6\% y una confiabilidad del 95\%. Se calculó un tamaño muestral de 139 participantes. Se realizó un muestreo secuencial estricto durante el período de estudio.

\section{Procedimiento}

Los datos fueron recolectados por los investigadores en un formato previamente diseñado y obtenidos de las historias clínicas de las pacientes atendidas en el servicio de partos del HSJD durante el período de estudio, revisando la historia clínica desde el ingreso hasta el alta.

Se registraron la edad, paridad, vía final del parto, complicaciones maternas tales como: ruptura uterina, desgarros vaginales y hemorragia posparto; también se registraron variables como peso, sexo y prueba de APGAR del recién nacido. Se calculó el puntaje predictor de parto vaginal que tenían al ingreso al hospital y se evaluó el desempeño de éste en dos puntos de corte: 4 y 8 puntos.

\section{Análisis}

Todos los datos se recopilaron y se procesaron en EXCEL 2003. Las variables categóricas se analizaron en proporciones y porcentajes. Las variables continuas se analizaron en promedios y rangos. Para evaluar el puntaje predictor se calculó la sensibilidad, especificidad y valores predictivos positivo y negativo; tomando como puntos de corte los puntajes de éxito para parto vaginal de 4 o más y 8 o más, ${ }^{28}$ y se compararon los dos puntos de corte con sus intervalos de confianza. Para las pruebas de significancia estadística se utilizó un intervalo de confianza del 95\%. Para determinar la capacidad predictiva del test se calcularon las razones de verosimilitud positiva (RVP) y negativa $(\mathrm{RVN})$ de ambos puntos de corte, utilizando las 
fórmulas: sensibilidad/(1-especificidad) para RVP y (1-sensibilidad)/especificidad para RVN, los cuales indican según el punto de corte evaluado cuánto es más probable el parto vaginal y la cesárea respectivamente.

\section{RESULTADOS}

Entre el 30 de mayo de 2007 y el 30 de mayo de 2008, hubo en el HSJD un total de 3842 nacidos vivos, de los cuales 1118 nacieron por cesárea, encontrándose una tasa de incidencia de cesárea del 29,1\%. En este período, 155 gestantes tenían antecedentes de cesárea. Se excluyeron 17 embarazadas; 3 por distocia pélvica, 8 por macrosomía fetal, 5 por presentación podálica y 1 por feto en situación transversa, quedando un total de 138 gestantes. En este grupo 28 de las gestantes tuvieron parto vaginal, para una incidencia del 20,3\%. 110 (79,7\%) fueron llevadas a segunda cesárea. Las principales indicaciones para esta segunda cesárea fueron: 100 (90,9\%) por el antecedente de cesárea previa, 3 $(2,7 \%)$ por sufrimiento fetal agudo, 3 (2,7\%) por trabajo de parto estacionario y $4(3,7 \%)$ por otras causas.

La mediana de la edad de estas 138 pacientes fue de 24 años, con un rango entre 16 a 38 años. La mediana de los embarazos previos fue 2 en un rango entre 2 y $10.14(10,1 \%)$ de ellas tenían un parto previo, 9 habían tenido un parto después de la primera cesárea y 5 un parto antes de la cesárea.
Entre las 110 gestantes que fueron llevadas a cesárea se encontró una dehiscencia del segmento uterino. Entre las pacientes que tuvieron parto vaginal no se presentaron complicaciones.

El promedio de peso de los recién nacidos, entre las mujeres que fueron a cesárea, fue 3312 gramos; 45 de sexo femenino, 65 de sexo masculino; 5 recién nacidos con asfixia perinatal leve y 1 con taquipnea transitoria del recién nacido. Entre las 28 mujeres que terminaron en parto vaginal, el promedio de peso fetal fue de 3225 gramos, y se presentó una taquipnea transitoria del recién nacido.

Al aplicar el puntaje predictor en aquellas gestantes con puntajes bajos, de 2 a 3, sólo 4 (5\%) terminaron en parto vaginal. En las pacientes con puntajes intermedios, de 4 a 7, 19 (36\%) tuvieron parto vaginal; y aquellas con 8 o más puntos, 5 $(83,3 \%)$ tuvieron parto vaginal. La sensibilidad para ambos puntos de corte fue mayor al $80 \%$ mientras la especificidad de puntaje de 4 o más no superó el 70\%. Los valores predictivos negativos estuvieron por encima del 90\% y ambos puntos de corte tuvieron bajos valores predictivos positivos (tablas 2 y 3).

$\mathrm{Al}$ calcular las razones de verosimilitud se halló que para el puntaje de 4 o más la RVP fue de 2,8; y la RVN fue de 0,2 . Y para el puntaje de 8 ó más la RVP fue de 4,8 y la RVN fue de 0,2.

\begin{tabular}{|l|c|c|c|c|}
\hline \multicolumn{7}{|c|}{ Tabla 2. Evaluación del puntaje predictor (valor $\geq 4$ puntos) } \\
\hline & & P. vaginal* & Cesárea & Total \\
\hline \multirow{2}{*}{$\begin{array}{l}\text { Puntaje } \\
\geq 4\end{array}$} & P. vaginal* & 24 & 34 & 58 \\
\hline & Cesárea & 4 & 76 & 80 \\
\hline
\end{tabular}

*Parto vaginal

Sensibilidad $=24 / 28=0,857=85,7 \%$, *IC95\% $(79,9-91,5)$

Especificidad $=76 / 110=0,691=69,1 \%, *$ IC $95 \%(61,4-76,8)$

Valor predictivo positivo (VPP) $=24 / 58=0,413=41,3 \%$, *IC95\% $(33,1-49,5)$

Valor predictivo negativo $(\mathrm{VPN})=76 / 80=0,95=95 \%, * \operatorname{IC} 95 \%(33,1-49,5)$

*IC: Intervalo de confianza 


\begin{tabular}{|c|c|c|c|c|}
\hline \multirow{4}{*}{$\begin{array}{l}\text { Puntaje } \\
\geq 8\end{array}$} & & P. vaginal* & Cesárea & Total \\
\hline & P. vaginal* & 5 & 23 & 28 \\
\hline & Cesárea & 1 & 109 & 110 \\
\hline & Total & 6 & 132 & 138 \\
\hline
\end{tabular}

*P. vaginal

Sensibilidad $=5 / 6=0,833=83,3 \%, * \operatorname{IC} 95 \%(77,1-89,5)$

Especificidad $=109 / 132=0,826=82,6 \%, * \operatorname{IC} 95 \%(76,3-88,9)$

Valor predictivo positivo $(\mathrm{VPP})=5 / 28=0,179=17,9 \%$, *IC95\% $(11,5-24,3)$

Valor predictivo negativo $(\mathrm{VPN})=109 / 110=0,991=99,1 \% * \operatorname{IC} 95 \%(97,5-100,1)$

*IC: Intervalo de confianza

\section{DISCUSIÓN}

El presente estudio, encontró una frecuencia de cesárea del 29,1\%, de parto vaginal posterior a cesárea del 20\% y una razón de verosimilitudes negativa del 0,20 para los dos puntos de corte del puntaje predictor de Flamm.

La frecuencia de cesárea encontrada fue similar a la descrita en América Latina, donde la tasa promedio es del 29\%, siendo las más altas en México 39\%, Brasil 36\%, Chile 30,1\%. En Colombia es del 27\%, y en Perú del 25\%. ${ }^{1-3}$

La Fundación Valle del Lili (Cali) reporta una tasa del 66,9\%. Según la Organización Mundial de la Salud (OMS) la tasa de cesárea no debería exceder el 15\%, pero estas tasas son variables en el mundo encontrándose en África 3,5\% en promedio, en Asia 15,9\%, siendo la más alta en China 40,2\%. Europa reporta un promedio del 19\%, el más alto en Italia con $36 \% .^{1,2}$

La tasa de parto después de una cesárea informada en nuestro estudio es una cifra baja comparada con los estudios en América Latina. Romero y colaboradores en su estudio en Argentina hallan un $78 \% ;{ }^{18}$ otro estudio realizado en México presenta un $49 \% ;{ }^{19}$ y estudios en Haití reportan tasas de parto del 73\%. ${ }^{21}$ Por otra parte, en un metaanálisis conducido por Guise JM et al, mostró que las tasas de parto alcanzan promedios del 60-82\%. ${ }^{15}$

En cuanto a las principales causas de cesárea en mujeres potencialmente candidatas a parto vaginal, nuestros resultados no son consistentes con los informados en la Fundación Valle del Lili (Cali) y en el Hospital Central de Asturias (España) donde el antecedente de cesárea previa fue del 32\% y $37,1 \%$, respectivamente, ${ }^{5,25}$ o en Argentina fue el sufrimiento fetal agudo $64 \% .^{20}$

En cuanto al puntaje descrito por Flamm, en el presente estudio se encontró que los porcentajes de éxito con el modelo de predicción fueron más bajos que los reportados por el autor. Sin embargo, estos datos coinciden con el estudio original en que las pacientes con mayor puntaje tuvieron una mayor probabilidad de tener un parto vaginal.

La principal preocupación en torno al parto vaginal en pacientes con cesárea previa, son las complicaciones. Una de las más temidas es la ruptura uterina. Se reporta que la ruptura uterina sintomática varía entre $0,2 \%$ y $0,7 \%$, muerte materna $0,02 \%$ e histerectomía $0,2 \% .^{7-10,15-18}$

Este es uno de los primeros estudios en nuestro medio que intenta evaluar los factores que favorecen el parto vaginal después de una cesárea previa, siendo ésta una fortaleza de esta investigación. El uso de las historias clínicas como única fuente de información puede afectar la calidad de algunos datos, ya que se percató de la existencia de algunos registros incompletos, lo cual puede ser una debilidad del estudio, a lo que se suma el tamaño de la muestra.

Los resultados de este estudio sugieren la importancia de realizar estudios de seguimiento 
que permitan evaluar los factores que podrían incrementar la probabilidad de parto vaginal después de una cesárea en nuestra población.

\section{CONCLUSIÓN}

En esta investigación se encuentra un alto porcentaje de cesárea en mujeres con una cesárea previa. Entre más alto el puntaje predictor al ingreso, se incrementa el porcentaje de parto vaginal. Sin embargo, su mayor utilidad está dada por la razón de verosimilitudes negativas.

\section{AGRADECIMIENTOS}

Por su colaboración, a las directivas y asistenciales del Hospital San Juan de Dios, a los estudiantes y profesores de la Universidad Santiago del Cali y a nuestras pacientes que son quienes le dan sentido a nuestra profesión.

\section{REFERENCIAS}

1. Tasas de cesáreas: análisis de los estimados regionales y nacionales. Rev Panam Salud Pública 2007;21:251.

2. World Health Organization. Programmes and projects. Making pregnancy safer. Country profiles on maternal and new born health. Visitado en 2011 May 19. Disponible en: http://www.who.int/making pregnancy_safer/countries/en/index.html

3. Casale RA. Niveles socioeconómicos y tasa de cesárea en Argentina. ¿Una inequidad en el acceso a la salud? Estudio ecológico. Revista de Obstetricia y Ginecología de Buenos Aires 2009;88:200-1

4. Echais F. Cesarea. Experiencia en el Hospital de Oxampampa. Ginecol obstet 2004;50:106-10.

5. Avanza MJ, Sosa TR, Giusti SA, Alegre C, Benitez A, Rivero MI. Frecuencia e indicaciones de cesárea en la población adolescente de un centro hospitalario. Revista Médica del Nordeste 2004;5:2-16.

6. Cobo E. Tasa de cesárea Fundación Clínica Valle del Lili. ¿Es posible reducirla? Colomb Med 1998;29:53-61.

7. Lydon-Rochelle M, Holt VL, Easterling TR, Martin DP. Risk of uterine rupture during labor among women with a prior cesarean delivery. N Engl J Med 2001;345:3-8.

8. Ricci P, Perucca E, Altamirano A. Nacimiento por parto vaginal después de una cesárea. Prueba de trabajo de parto versus cesárea electiva a repetición. Rotura uterina, una complicación posible. Rev Chil Obstet Ginecol 2003;68:523-8.

9. Rosen MG; Dickinson JC; Westhoff CL. Vaginal birth after cesarean: a meta-analysis of morbidity and mortality. Obstet Gynecol 1991;77:465-70.

10. Mozurkewich EL, Hutton EK. Elective repeat cesarean delivery versus trial of labor: a meta-analysis of the literature from 1989 to 1999 . Am J Obstet Gynecol 2000;183:1187-97.

11. Diaz H, Cusati R, Carrillo H et al. Parto insturmental en cesarea anterior: importancia del intervalo inteparto. Rev Obstet Ginecol Venez 2007;67:79-86.

12. Ortiz Lee C, de Marcos López N. ¿Estamos preparados para enfrentar el parto vaginal posterior a cesárea? Rev Cubana Obstet Ginecol 1998;24:65-8.

13. Ortiz Lee C, de Marcos López N. Parto vaginal pos cesarea. Primeros casos en Cuba. Rev Cubana Obstet Ginecol 1998:24:117-21.

14. ACOG Practice Bulletin Clinical management Guidelines for Obstetrician-Gynecologists. Vaginal Birth After Previous Cesarean Delivery. Number 54; 2004.

15. Guise JM, Berlin M, McDonagh M, Osteweil P, Chan B, Helfand M. Safety of vaginal birth after cesarean: a systematic review. Obstet Gynecol 2004;103:420-9.

16. Eden KB, Hashima JN, Osterweil P, Nygren P, Guise JM. Childbirth preferences after cesarean birth: a review of the evidence. Birth 2004;31:49-60.

17. Landon MB, Hauth JZ, Leveno KJ, Spong CY, Leindecker S, et al. Maternal and Perinatal Outcomes Associated with a Trial of Labor after Cesarean Delivery. N Engl J Med 2004.352:2581-9.

18. Romero A, Arce R, Lind S, Bazan C. prueba de trabado de parto en pacientes con antecedente de cesárea previa. Revista de Posgrado de VIa Cátedra de Medicina 2004:12-6.

19. Sánchez R, Ortiz HI, Chávez A. Parto vaginal en pacientes con antecedente de cesárea previa. Rev Fac Med UNAM 2003;46:28-31.

20. Mizunoya F, Nakata M, Kondo T, Yamashita S, Inoue S. Management of vaginal birth after cesarean. J Obstet Gynaecol Res 2002;28:240-4.

21. Vergara Reina R, Acosta Pérez M. Experiencias en el parto vaginal poscesárea en un hospital haitiano. Rev Cubana Obstet Ginecol 2003;29(1).

22. Grinstead J, Grobman W. Induction of labor after one prior cesarean: Predictors of vaginal delivery. Obstet Gynecol 2004;103:534-8. 
23. Rodríguez E, Escudero A, Gaite M, Suárez M.T. Vía del parto tras cesárea anterior: factores asociados. Prog Obstet Ginecol 2003;46:250-6.

24. Hashima JN, Eden KB, Osterweil P, Nygren P, Guise JM. Predicting vaginal birth after cesarean delivery: A review of prognostic factors and screening tools. Am J Obste and Gynecol 2004;190:547-55.

25. Guise JM, Eden K, Emeis C, Denman MA, Marshall N, Fu RR, et al. Vaginal birth after cesarean: new insights. Evid Rep Technol Asses (Full Rep) 2007;2010:1-397.
26. Cecatti JG, Pires HM, Faúndes A, Duarte Osis MJ. Factors associated with vaginal birth after previous cesarean section in Brazilian women. Rev Panam Salud Pública 2005;18:107-13.

27. Ebell MH. Predicting the likelihood of successful vagina birth after cesarean delivery. Am Fam Physician 2007;76:1192-4.

28. Flamm BL, Geiger AM. Vaginal birth after cesarean delivery: an admission scoring system. Obstet Gynecol 1997;90:907-10. 